\title{
Millau, Creissels, Castelnau-Pegayrols
}

\section{Christophe Saint-Pierre}

\section{CpenEdition \\ Journals}

Édition électronique

URL : http://journals.openedition.org/adlfi/17240

ISSN : 2114-0502

Éditeur

Ministère de la culture

Référence électronique

Christophe Saint-Pierre, " Millau, Creissels, Castelnau-Pegayrols », ADLFI. Archéologie de la France Informations [En ligne], Midi-Pyrénées, mis en ligne le 03 mai 2016, consulté le 03 mai 2019. URL

http://journals.openedition.org/adlfi/17240

Ce document a été généré automatiquement le 3 mai 2019.

(C) Ministère de la Culture et de la Communication, CNRS 


\title{
Millau, Creissels, Castelnau- Pegayrols
}

\author{
Christophe Saint-Pierre
}

Lien Atlas (MCC) :

http://atlas.patrimoines.culture.fr/atlas/trunk/index.php?

ap_theme=DOM_2.01.02\&ap_bbox=2.991;44.016;3.245;44.180

1 Dans le cadre de l'approche territoriale des Grands Causses à la fin de l'Antiquité et au haut Moyen Âge, il convenait de reprendre l'étude de plusieurs sites identifiés, parfois partiellement fouillés, dans la région millavoise, afin de mettre ce réseau de sites en perspective avec l'occupation de hauteur de la Granède pour la même période.

2 L'objet de cette prospection inventaire était de relocaliser avec précisions ces gisements, et d'observer la présence de vestiges visibles.

\section{Millau}

\section{Le Serre du Cocut et l'Angle}

3 Localisés sur le plateau du Larzac, ces deux sites sont implantés sur le versant d'une dépression, ouverte vers le sud, et dont le fond est occupé en partie par un champ cultivé. Ils ont été identifiés par Rémi Azémar lors de ses travaux sur la nécropole mégalithique voisine de Saint-Martin-du-Larzac.

4 Plusieurs structures maçonnées en pierre sèche, fortement arasées, sont regroupées en trois points autour du champ cultivé. Le plus souvent de plan rectangulaire, elles se rapprochent fortement des structures pastorales étudiées par Laurent Fau sur le plateau de l'Aubrac. La céramique collectée dans le champ montre un horizon chronologique compris entre le $\mathrm{V}^{\mathrm{e}}$ et le VII ${ }^{\mathrm{e}} \mathrm{s}$. apr. J.-C. 
Ces premières observations, recoupant celles déjà faites par Rémi Azémar, nous invitent à voir ici un habitat groupé à vocation agro-pastorale du début du haut Moyen Âge.

\section{Fabrègues/Combe Longue}

6 Toujours sur le plateau du Larzac, localisé entre les lieux-dits de Combebren et de la Blaquière, le site de Fabrègues/Combe Longue est connu suite à des fouilles réalisées en 1940. Une dizaine de sépultures auraient alors été fouillées. Le mobilier a été déposé au Musée de Millau.

7 Il convenait pour nous de relocaliser cette nécropole et de vérifier la conservation éventuelle des coffres mis au jour en 1940.

8 L'emplacement a bien été retrouvé et plusieurs structures sont encore visibles. L'information majeure retenue sur ce site est la présence à la surface du sol de plusieurs amas de petites pierres, sur une faible hauteur, ne correspondant manifestement pas à l'épierrement d'un champ. Il est probable que cela soit le marquage de sépultures encore intactes, dispositif de signalisation que nous n'avons pas pu mettre fermement en évidence sur le site de la Granède.

\section{Puech d'Andan}

9 Au nord de l'agglomération de Millau, la butte du Puech d'Andan présente deux petits plateaux dont les points culminants sont compris entre $872 \mathrm{~m}$ et $883 \mathrm{~m}$ NGF. Le plateau oriental se prolonge en direction de Millau par une pointe rocheuse dont l'extrémité se situe à $823 \mathrm{~m}$ d'altitude.

10 Dès 1921, l'érudit Albert Carrière signale, sur l'indication de l'abbé Hermet, la présence de deux « caps barrés » sur le Puech d'Andan. Ultérieurement, des fragments de céramiques dérivées de sigillées paléochrétiennes auraient été trouvés sur le cap barré du plateau oriental surplombant Millau. La prospection organisée en 2013 visait à retrouver les vestiges de ce cap barré, d'accès difficile et totalement masqué par la végétation, et à montrer sa contemporanéité avec la Granède qui lui fait face.

11 Plusieurs segments de murs ont effectivement été retrouvés, parfois observés sur $2 \mathrm{~m}$ de hauteur. Construits en moellons de calcaire, équarris et non liés au mortier, ils condamnent l'accès à la plateforme. Malheureusement, aucun indice ne nous autorise à proposer une datation pour ces dispositifs.

\section{Creissels}

\section{Le Plateau de France}

12 Un seul site a été retenu dans le cadre de cette prospection sur la commune de Creissels, mitoyenne avec l'agglomération millavoise. La revue Gallia mentionne en 1974 la découverte, effectuée l'année précédente, de trois sépultures datées du vII ${ }^{\mathrm{e}} \mathrm{s}$. apr. J.-C. dans une petite grotte située à l'extrémité ouest du Plateau de France.

L'objectif de la prospection menée cette année était de rechercher la présence d'habitat possible sur le plateau lui-même, pouvant être en lien avec les sépultures. 
14 Aucune information n'a pu être mise en évidence. La prospection du pied de falaises autour du site, et en particulier vers l'ouest, a permis de localiser plusieurs petites grottes ou cavités. Deux d'entre elles renfermaient des ossements humains en position secondaire déposés à même le sol. Une datation par radiocarbone a pu être effectuée pour chacun des dépôts. Les résultats obtenus sont compris entre 2344 et 2141 av. J.-C. pour l'un (Date $14^{\mathrm{C}}$ calibrée : $2344 \mathrm{cal} \mathrm{BC}-2141 \mathrm{cal} \mathrm{BC}$ courbe de calibration «IntCal04", Reimer et al., 2004; Radiocarbon, 46. $\left.\mathrm{N}^{\circ} \mathrm{ETH}-54036\right)$ et 2572 et 2349 av. J.-C. pour l'autre (Date $14^{\mathrm{C}}$ calibrée : $2572 \mathrm{cal} \mathrm{BC}-2349 \mathrm{cal} \mathrm{BC}$ courbe de calibration «IntCal04 », Reimer et al., 2004, Radiocarbon, 46. $\left.\mathrm{N}^{\circ} \mathrm{ETH}-54037\right)$.

\section{Castelnau-Pégayrols}

\section{Navas}

15 Seul le site de la nécropole de Navas a été prospecté sur cette commune. Comme le site de Fabrègues/Combe Longue, il pouvait nous aider à mieux comprendre l'organisation d'un espace funéraire du haut Moyen Âge et éclairer ainsi celui de la Granède sur lequel l'effondrement des murs du bâtiment ecclésial nous avait privés d'informations. Nous devons la fouille d'une vingtaine de sépultures de cette nécropole à Louis Balsan au début des années 1930. Plusieurs d'entre elles ont fourni du mobilier permettant d'attribuer cet ensemble aux $\mathrm{VI}^{\mathrm{e}}-\mathrm{VII}^{\mathrm{e}} \mathrm{s}$. apr. J-C.

Comme pour la nécropole de Fabrègues/Combe Longue, nous avons pu retrouver les coffres en dalles de calcaire des sépultures et, cette fois encore, plusieurs petits amas de pierre de forme oblongue et respectant l'orientation des sépultures fouillées étaient encore visibles.

Bien que modeste, et sur la base de gisements déjà connus, mais pas toujours localisés avec précision, cette campagne de prospection inventaire nous aura permis de relever plusieurs informations utiles dans le cadre des recherches menées sur le site de hauteur de la Granède.

18 Il s'agit en particulier du type de marquage des sépultures et de la forme de l'habitat à caractère agropastoral, probablement présent aux abords du site de la Granède si l'on prend en compte les informations données par les prospections sur les terres de la ferme de l'Hôpital.

INDEX

Index chronologique : Moyen Âge

Index géographique : Midi-Pyrénées, Aveyron (12), Millau, Creissels, Castelnau-Pegayrols

Mots-clés : céramique, habitat, nécropole, coffre, ossement, amas

operation Prospection inventaire (PI) 
AUTEURS

CHRISTOPHE SAINT-PIERRE

COL 12 